\title{
Examining the Impact of Adverse Weather on Travel Time Reliability of Urban Corridors in Shanghai
}

\author{
Yajie Zou $\mathbb{D}$, ${ }^{1}$ Ting Zhu $\mathbb{D},{ }^{1}$ Yifan Xie $\mathbb{D},{ }^{1}$ Linbo Li $\mathbb{D},{ }^{1}$ and Ying Chen $\mathbb{D}^{2}$ \\ ${ }^{1}$ Key Laboratory of Road and Traffic Engineering of Ministry of Education, Tongji University, Shanghai 201804, China \\ ${ }^{2}$ School of Traffic and Transportation Engineering, Changsha University of Science \& Technology, Changsha 410114, China \\ Correspondence should be addressed to Linbo Li; llinbo@tongji.edu.cn
}

Received 16 July 2020; Revised 14 November 2020; Accepted 24 November 2020; Published 17 December 2020

Academic Editor: Jinjun Tang

Copyright (c) 2020 Yajie Zou et al. This is an open access article distributed under the Creative Commons Attribution License, which permits unrestricted use, distribution, and reproduction in any medium, provided the original work is properly cited.

Travel time reliability (TTR) is widely used to evaluate transportation system performance. Adverse weather condition is an important factor for affecting TTR, which can cause traffic congestions and crashes. Considering the traffic characteristics under different traffic conditions, it is necessary to explore the impact of adverse weather on TTR under different conditions. This study conducted an empirical travel time analysis using traffic data and weather data collected on Yanan corridor in Shanghai. The travel time distributions were analysed under different roadway types, weather, and time of day. Four typical scenarios (i.e., peak hours and off-peak hours on elevated expressway, peak hours and off-peak hours on arterial road) were considered in the TTR analysis. Four measures were calculated to evaluate the impact of adverse weather on TTR. The results indicated that the lognormal distribution is preferred for describing the travel time data. Compared with off-peak hours, the impact of adverse weather is more significant for peak hours. The travel time variability, buffer time index, misery index, and frequency of congestion increased by an average of $29 \%, 19 \%, 22 \%$, and $63 \%$, respectively, under the adverse weather condition. The findings in this study are useful for transportation management agencies to design traffic control strategies when adverse weather occurs.

\section{Introduction}

Travel time reliability (TTR) is an important measure of traffic condition and is widely used to evaluate transportation system performance. The Federal Highway Administration (FHWA) [1] formally defined the TTR as "the consistency or dependability in travel times, as measured from day-to-day and/or across different times of the day." The TTR can be used to represent the probability of on-time arrival; thus, it is a key factor for roadway users to make decisions on travel routes and departure time. A survey conducted by Abdel-Aty et al. [2] shows that about 54\% respondents consider TTR as an important factor for choosing commute routes. Unreliable travel times can cause significant inconvenience to roadway users and result in high time and monetary losses. Thus, some studies analysed the monetary value of TTR [3-5]. For instance, Lam [5] conducted a study in California and found that the value of travel time is $\$ 22.47$ per hour (as 68 percentile of average salary at that time), while the value of TTR is $\$ 31.16$ per hour (as 95 percentile of average salary at that time). Meanwhile, there are several studies focusing on the relationship between TTR and traffic safety, and it is confirmed that the reduction of travel time reliability would lead to severe traffic collisions and crashes [6-10]. In recent years, some studies have found that improving travel time reliability can help reduce fuel consumption and emissions by avoiding stopand-go movements of vehicles $[11,12]$. Thus, improving the TTR on the entire road network can be essential and valuable.

To accurately evaluate the travel time reliability on transportation facilities, substantial studies focus on developing appropriate TTR measures. Lomax et al. [13] comprehensively summarized the common TTR measures and categorized them into four types: statistical range methods, buffer time methods, tardy-trips measures, and probabilistic measures. Table 1 provides a summary of the widely used TTR measures in recent years. Gao et al. [18] 
TABLE 1: Summary of widely used TTR measures.

\begin{tabular}{|c|c|c|}
\hline Category & Measure & Equation \\
\hline \multirow{4}{*}{ Statistical range methods } & Coefficient of deviation [14] & Standard deviation/average travel time \\
\hline & Travel time variability [15] & TT90-TT10 \\
\hline & Skew of travel time distribution [16] & TT90 - TT50/TT50 - TT 10 \\
\hline & Width of travel time distribution [16] & TT90 - TT10/TT50 \\
\hline \multirow{4}{*}{ Buffer time methods } & Planning time index $[1]$ & 95th percentile travel time/free flow travel time \\
\hline & Buffer time index [13] & (95th percentile travel rate/free flow travel rate) -1 \\
\hline & Travel time index [17] & Average travel time/free flow travel time \\
\hline & Misery index [17] & (Average travel rate (top $20 \%$ trips)/average travel rate) -1 \\
\hline Tardy-trip measures & On-time arrival [17] & $\begin{array}{l}\text { Percent of trips with travel time less than } 110 \% \text { or } 125 \% \text { of the } \\
\text { median travel time }\end{array}$ \\
\hline Probabilistic measures & Frequency of congestion [1] & Frequency of trips experiencing traffic congestion \\
\hline
\end{tabular}

investigated the value of several TTR measures under different traffic conditions, and they found that the volatility trends of them were similar, while the magnitude of change values of Frequency of Congestion was significant. However, TTR measures demonstrate various characteristics. For example, the probabilistic measures can be used to calculate the probability of arrival before a certain time, which are user-friendly and can help travelers to plan trips. In recent years, TTR has been widely used to evaluate traffic conditions and help optimize transportation management based on these TTR measures [19-23]. For instance, CedilloCampos et al. [23] conducted a study to assess freight fluidity of transportation systems with the help of TTR measures (i.e., percentiles of travel time, planning time index, buffer time index, and skew and range of travel).

Knowledge about which factors affect TTR and how they influence TTR can help improve the travel time reliability. Based on previous studies, the factors affecting TTR can be classified into two categories [24], factors causing demand variation (such as season, time of day, and weather), and factors causing supply variation (such as traffic incident, road geometry, road work, and weather). It can be seen that weather influences the TTR on two ways. Several studies evaluated the impact of adverse weather on travel time reliability, and it is demonstrated that the adverse weather has negative effects on TTR above a certain critical inflow [15, 25-27]. However, most studies among them focus on freeways, and very few studies comprehensively evaluate the impact of adverse weather on urban corridor (especially arterial roadway) travel time reliability. Travel time and travel time reliability of different highway types are supposed to exhibit diverse patterns due to the difference of traffic characteristics. Thus, there is a need to explore the difference of adverse weather's impacts on TTR of urban corridor. In addition, the analysis of the impact of adverse weather on TTR of urban corridor under different traffic conditions can be useful for traffic management. The study has two primary objectives. The first objective is to explore the characteristics of urban corridor travel time. The second objective is to quantitatively estimate the impact of adverse weather on TTR under different conditions.

The rest of this paper is organized as follows. The second section introduces the single-mode distributions and travel time reliability measures used in this study to explore the travel time characteristics of the urban corridor. In Section 3, the data preparation procedures are introduced. Section 4 illustrates the results and compares the impact of adverse weather on TTR under different conditions. Finally, Section 5 provides conclusions and identifies future directions for this research.

\section{Methodology}

2.1. Travel Time Distribution. Travel time distribution (TTD) describes the shape of travel time under different travel conditions. It provides a straightforward and visualized tool for modelling the average travel time and travel time variability. In this study, five commonly used single-mode distribution types are considered to fit travel time data, including Weibull distribution, Gamma distribution, Normal distribution, Lognormal distribution, and Log-logistic distribution. The details of five distributions are given in Table 2 .

Based on the travel time data, the parameters of each distribution are estimated using software $\mathrm{R}$. In order to compare the goodness-of-fit results of different distributions, the information criteria are adopted to select the better fit one. Information criteria mainly refer to Akaike information criterion (AIC) and Bayesian information criterion (BIC) [28].

AIC is a measure to compare relative quality of different models. Based on the principle of asymptotically unbiased estimation, Akaike proposed an exact estimation algorithm, as shown in

$$
\mathrm{AIC}=2 k-2 \ln (L),
$$

where $k=$ the number of parameters and $L=$ the value of likelihood function.

Analogous to AIC, BIC introduces a penalty term associated with the parameter number to avoid the occurrence of over fitting problem. Thus, only AIC is calculated to evaluate the performance of five distributions. A smaller AIC value indicates better fitting performance.

2.2. Travel Time Reliability Measures. As aforementioned, there are several measures to describe travel time reliability. In this study, four widely used measures are adopted to evaluate the TTR, and they are travel time variability (TTV), 
TABle 2: Description of five distributions.

\begin{tabular}{lcc}
\hline & Function & Parameters \\
\hline Weibull distribution & $f(y \mid \gamma, \beta)=(\gamma / \beta) y^{\gamma-1} e^{-y^{\gamma} / \beta}$ & $\gamma, \beta$ \\
Gamma distribution & $f(x \mid \alpha, \beta)=\left(1 / \Gamma(\alpha) \beta^{\alpha}\right) x^{\alpha-1} e^{-x / \beta}$ & $\alpha, \beta$ \\
Normal distribution & $f\left(x \mid \mu, \sigma^{2}\right)=(1 / \sqrt{2 \pi \sigma}) e^{-(x-\mu)^{2} / 2 \sigma^{2}}$ & $\mu, \sigma^{2}$ \\
Lognormal distribution & $f\left(x \mid \mu, \sigma^{2}\right)=(1 / \sqrt{2 \pi \sigma} x) e^{-(\log x-\mu)^{2} / 2 \sigma^{2}}$ & $\mu, \sigma^{2}$ \\
Log-logistic distribution & $f(x \mid \mu, \sigma)=(1 / \sigma) e^{(x-\mu / \sigma)} /\left(1+e^{(x-\mu / \sigma)}\right)^{2}$ & $\mu, \sigma$ \\
\hline
\end{tabular}

buffer time index (BTI), misery index (MI), and frequency of congestion (FOC).

Travel time variability (TTV) is a statistical range method, representing the difference between the $90^{\text {th }}$ and $10^{\text {th }}$ percentile travel time, as shown in (2). Some previous studies found that the statistical properties of TTV can be more robust than moment-based measures (such as mean and deviation) $[29,30]$ :

$$
T T V=T T 90-T T 10,
$$

where TT90 $=90^{\text {th }}$ percentile travel time ( $\mathrm{min}$ ) and TT10 $=10^{\text {th }}$ percentile travel time $(\mathrm{min})$.

Buffer time index (BTI) is a typical buffer time method, proposed by Lomax et al. [13]. It is calculated by (3), which reflects the percentage of extra travel time that most travelers need to add to the mean travel time in order to arrive on time. This index depends on the travel rate, which is calculated as the travel time divided by the distance:

$$
\mathrm{BTI}=\frac{\text { 95th percentile travel rate }- \text { average travel rate }}{\text { average travel rate }},
$$

where the $95^{\text {th }}$ percentile travel rate and average travel rate are in minutes per $\mathrm{km}$.

Misery index (MI) proposed by Lomax is a tardy-trip measure. Similar to BTI, it is related to travel rate as well as the worst trips. This TTR measure can represent the traffic conditions and identify the roadway segments experiencing congestion. MI is defined as the ratio of the difference between the average travel rate for the longest $20 \%$ of trips and average travel rate to the average travel rate, and the equation is provided as follows:

$\mathrm{MI}=\frac{\text { average travel rate of the top } 20 \% \text { trips }- \text { average travel rate }}{\text { average travel rate }}$,

where two average travel rates are in minutes per $\mathrm{km}$.

Frequency of congestion (FOC) represents the frequency of trips exceeding a threshold value, which is a probabilistic measure. This research assumed that the traffic congestion occurs when the travel time is higher than twice the free-flow travel time. The free-flow speed of each route is obtained as the $85^{\text {th }}$ percentile speed during overnight hours $(10$ p.m. to 5 a.m.) $[9,31,32]$. Note that the free-flow travel time of each route is inversely proportional to free-flow speed; thus, the free-flow travel time is defined as the $15^{\text {th }}$ percentile travel time during overnight hours. The FOC can be calculated by using the following:

$$
\begin{aligned}
& G(p)=2 G(0.15), \\
& F O C=1-p,
\end{aligned}
$$

where $p$ is the frequency of travel time higher than twice the free-flow travel time.

\section{Data Description}

Two datasets are adopted in this study to investigate the impact of adverse weather on TTR of urban corridor. The traffic data collected from the Gaode Map website are used to generate travel times, while the weather data associated with the travel times are collected from China Meteorological Administration.

3.1. Description of Study Site. Four routes along Yanan elevated expressway and arterial road are selected for this study. The Yanan elevated expressway has six lanes and connects the Hongqiao International Airport to the Bund of Shanghai, as shown in Figure 1. The selected study section of the elevated expressway is $14.32 \mathrm{~km}$ long and covers the eastbound and westbound directions. The Yanan elevated expressway is basically constructed along Yanan West Road, Yanan Middle Road, and Yanan East Road. Thus, the corresponding arterial road is around $14.32 \mathrm{~km}$ long as well, and traffic data for both directions are collected.

The main reasons for choosing these four routes are summarized as follows. Previous studies suggest that adverse weather has minor effect on TTR for free flow traffic condition but can significantly affect TTR at high traffic flow level $[15,30]$. In addition, the Yanan elevated expressway is a main corridor in Shanghai and experiences heavy traffic during the peak hours.

3.2. Description of Travel Time Data. The Gaode Map divides one route into fixed consecutive sections of the specified length and sets five congestion levels. From the end of the road, it records the congestion levels of road sections. Adjacent road sections with the same congestion level converge on one piece of data, recording starting point, ending point, direction, and average speed. The raw traffic data are collected every 4 minutes. Traffic data from October 2019 to February 2020 are used in this study, which contain the following information: road ID, driving direction, congestion level, speed, timestamp and so on. This study aims to analyse the differences of adverse weather's impact on TTR during peak and off-peak hours. Thus, the traffic data collected from $6: 00 \mathrm{a} . \mathrm{m}$. to $22: 00 \mathrm{p} . \mathrm{m}$. on weekdays are utilized. 


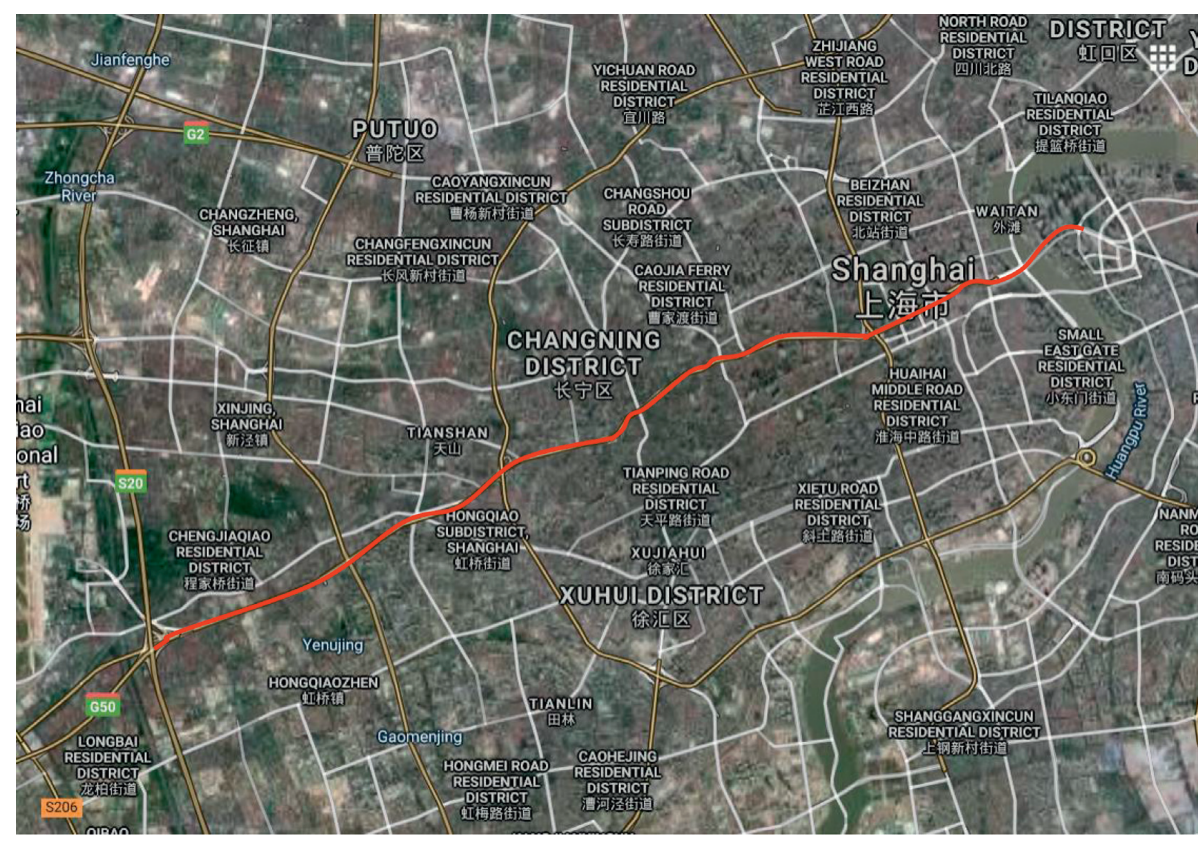

FIgURE 1: Map of Yanan corridor in Shanghai, China.

After excluding the traffic data with invalid or missing essential information, route-level travel times are obtained through several steps. First, calculate single section travel time based on each piece of data. Based on the starting and ending points, the length of section is determined. Then, the single section travel time is calculated by dividing the length of section by average speed. Second, route-level travel times are taken by summing all sections' travel time.

3.3. Description of Weather Data. The historical weather data of four districts (Huangpu, Changning, Jing'an, and Baoshan districts) within the study time period are obtained. The raw weather data classify weather conditions into four groups, including normal, overcast, mostly cloudy, and rain. According to previous studies, it is demonstrated that only adverse weather can cause a significant impact on traffic conditions [15]. Thus, "overcast" and "mostly cloudy" are classified into "normal" in this study due to no obvious influence on travel times. Meanwhile, this study tried to classify "rain" into more types (e.g., snow, ice, and fog) based on detailed weather information such as temperature, visibility, and humidity. In order to ensure the acceptable sample size, this study combined the adverse weather together as "rain" due to the rarity of other adverse weather types. Therefore, the weather variables are categorized as "normal weather" and "adverse weather."

Two datasets are matched based on timestamp. However, the weather data are collected every 20 minutes, while the travel time data are aggregated into 4-minute intervals. In order to merge the link travel time data with weather data, the weather data are expanded by filling the absence as the weather condition of the most recent timestamp. The Yanan elevated expressway covers four districts. To improve the accuracy of analysis, this study only selects the data when the weather conditions of four districts are the same for research.

The average travel time (in 15-minute period) of each route under different weather conditions were calculated and shown in Figure 2. Note that in Figure 2, the horizontal axis denotes the day from 6:00 to 22:00 with 15 minutes as a time interval, and the vertical axis represents the mean of the travel times during desired time period. Previous studies $[15,33]$ concluded that time intervals between 10 and 15 minutes are suitable for analysing traffic conditions. For a better visual effect, this paper adopted a 15-minute time interval to draw scatter plots.

According to Figure 2, there are several points worth mentioning. First, the difference of the travel time between normal weather condition and adverse weather condition is remarkable during the peak time. This finding indicates that the adverse weather has significant impact on travel time only when traffic volume is above a certain value. Second, there is no a.m. peak hour characteristic of travel time in Figure 2(b). This can be probably explained by the influence of commuting resulting in the difference of traffic volume between driving directions at the same time period. Thus, it is also worth exploring the difference of TTR with respect to driving direction. Third, the travel times of elevated expressway are high at noon (13:00-15:00). The main reason for this could be that only vehicles with Shanghai license plate are permitted to enter Yanan elevated expressway after 15:00. Therefore, before that time, a majority of cars without a Shanghai license plate leave the city centre through the elevated expressway.

For analysing the impact of adverse weather on TTR under different traffic conditions, two typical time periods are selected as peak hours, that is, AM peak hours (7:30-9: $00)$ and PM peak hours (16:30-18:30). 


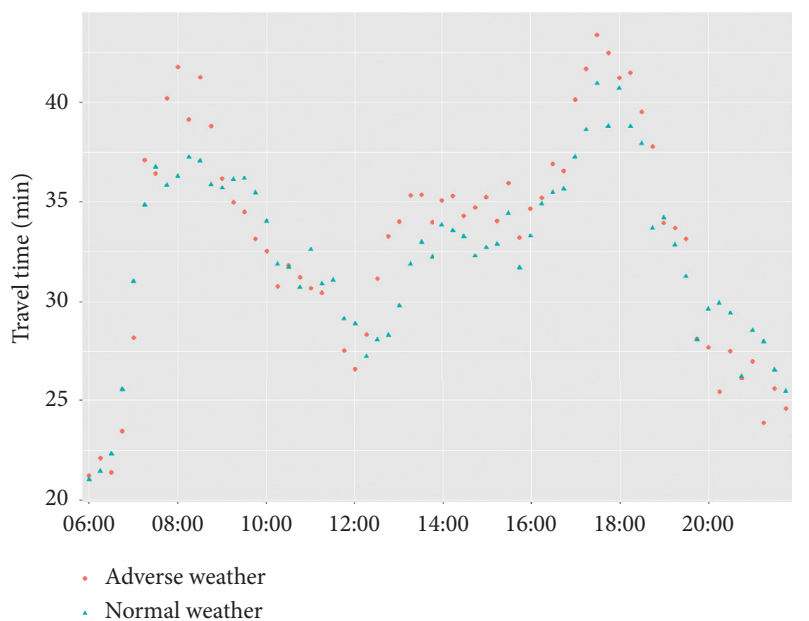

(a)

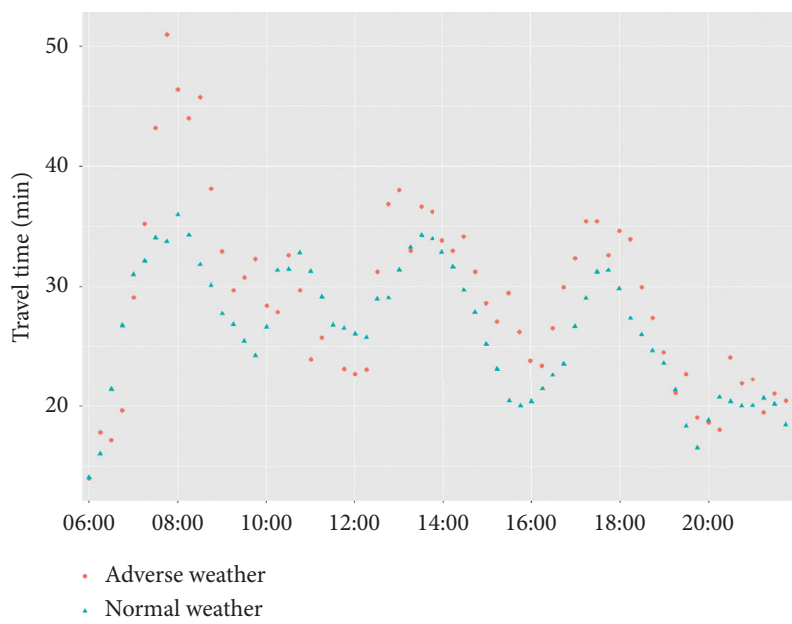

(c)

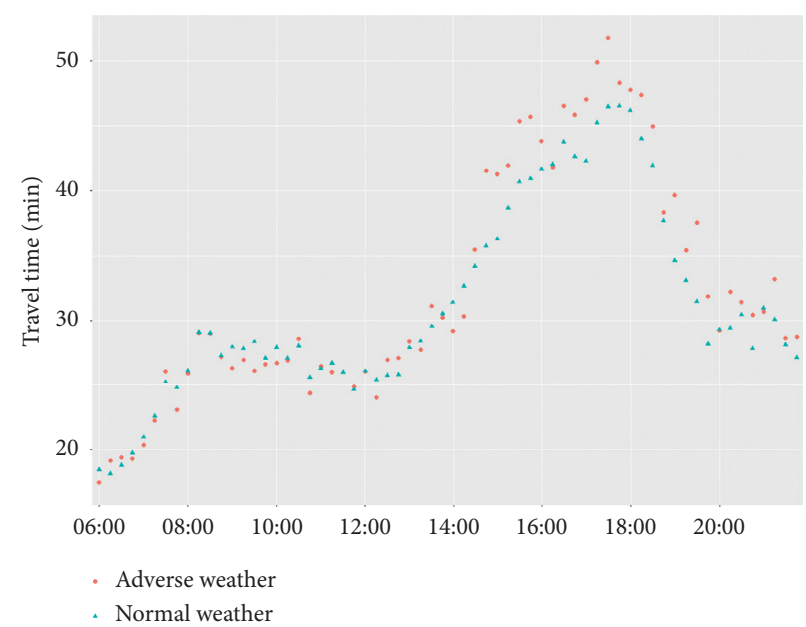

(b)

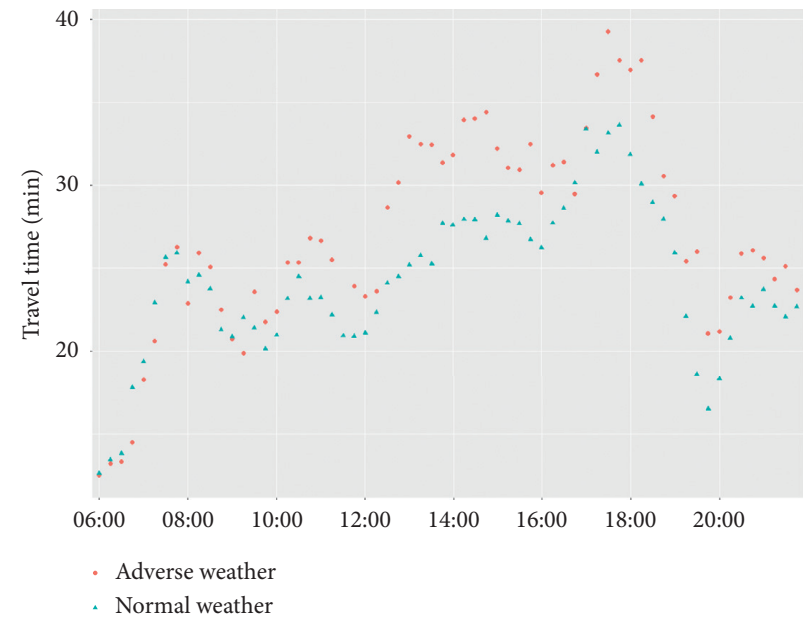

(d)

FIgURE 2: Travel time of each route under different weather conditions. (a) Yanan arterial road Eastbound. (b) Yanan arterial road Westbound. (c) Yanan elevated expressway Eastbound and (d) Yanan elevated expressway Westbound.

\section{Results and Discussion}

4.1. Travel Time Distribution Analysis. Five distribution types were fitted with the observed travel time data for each route. In this paper, the best-fit distribution is defined as the one with the minimum AIC value. Figure 3 shows the probability density of the best-fit distribution and the observed travel time data for each route. The legend displays the distribution type and parameters of the best-fit distribution. It can be found that the lognormal distribution provides the best model fit for all routes. According to the density curves of observed travel time data, the average travel time on arterial road is higher than that of expressway.

To illustrate the travel time distribution under different weather conditions, the travel time data are divided into four categories: elevated expressway data under adverse weather and normal weather conditions, arterial road data under adverse weather and normal weather conditions. Then, five distribution types are fitted with the travel time data.
Figure 4 shows the probability density of the best fitting distribution and the observed travel time data under different weather conditions. Based on Figure 4, it can be found that the lognormal distribution fits better than other distributions.

To illustrate the travel time distribution under different time periods, the travel time data are divided into four categories. Then, five distribution types are fitted with the travel time data for each dataset. Figure 5 shows the probability density of the best-fit distribution and the observed travel time data during different time periods. The results show that the lognormal distribution fits best for most scenarios, except the arterial road data observed during peak hours.

4.2. TTR Analysis Results for Different Traffic Directions. To investigate the travel time reliability of four studied routes, the TTR measures of each route are calculated, as shown in Figure 6. The travel time on westbound Yanan arterial road is less reliable. The BTI and MI of westbound 


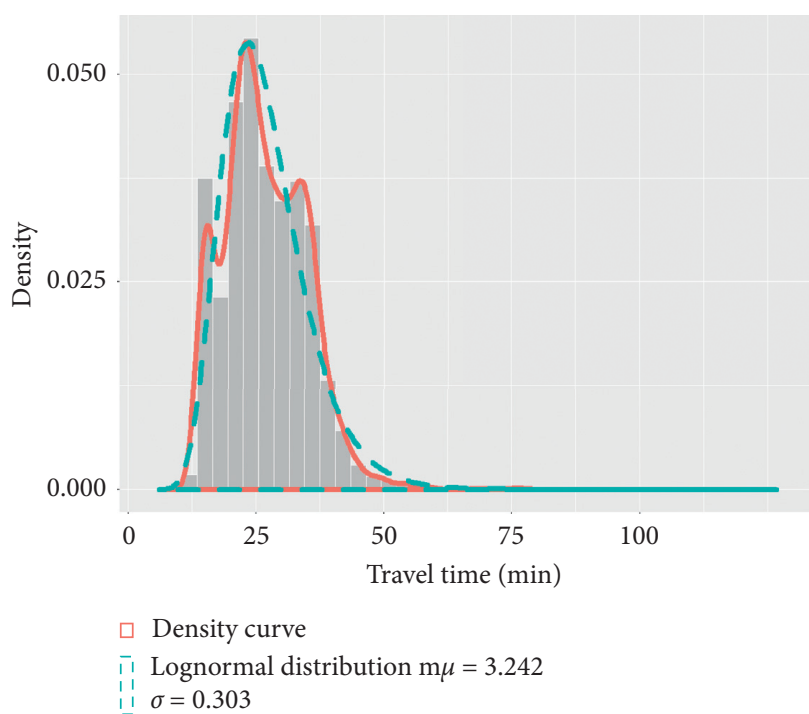

(a)

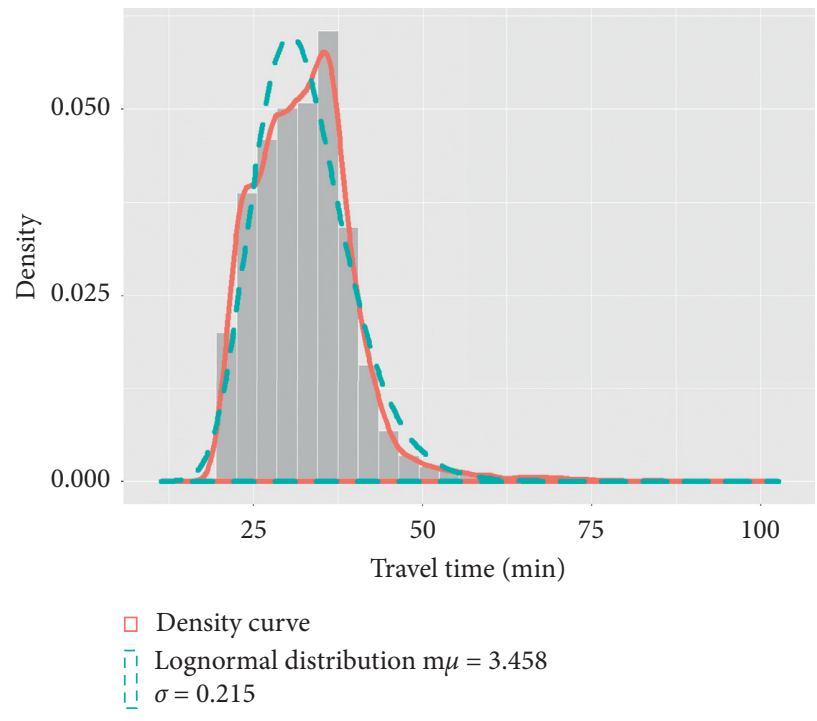

(c)

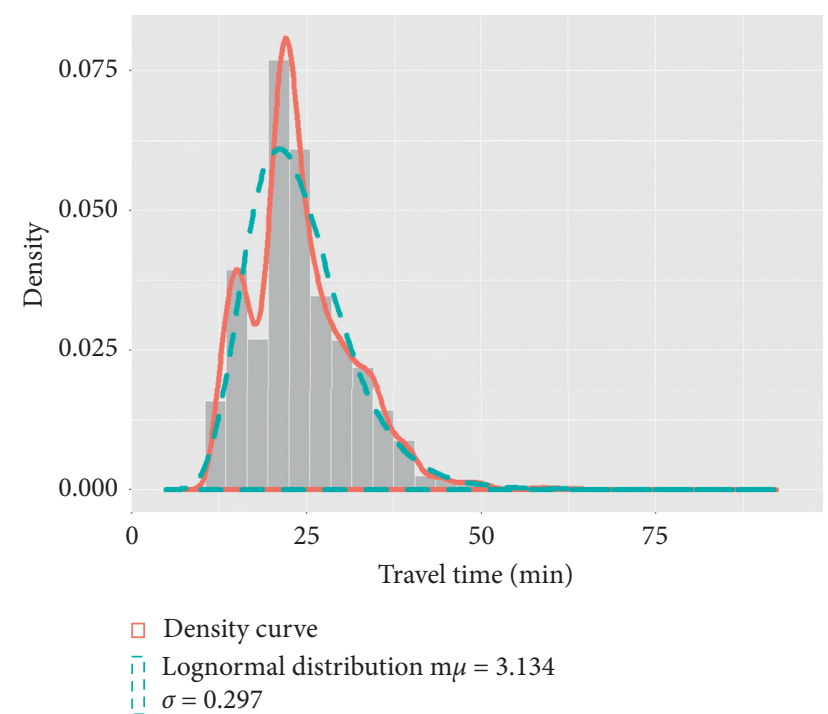

(b)

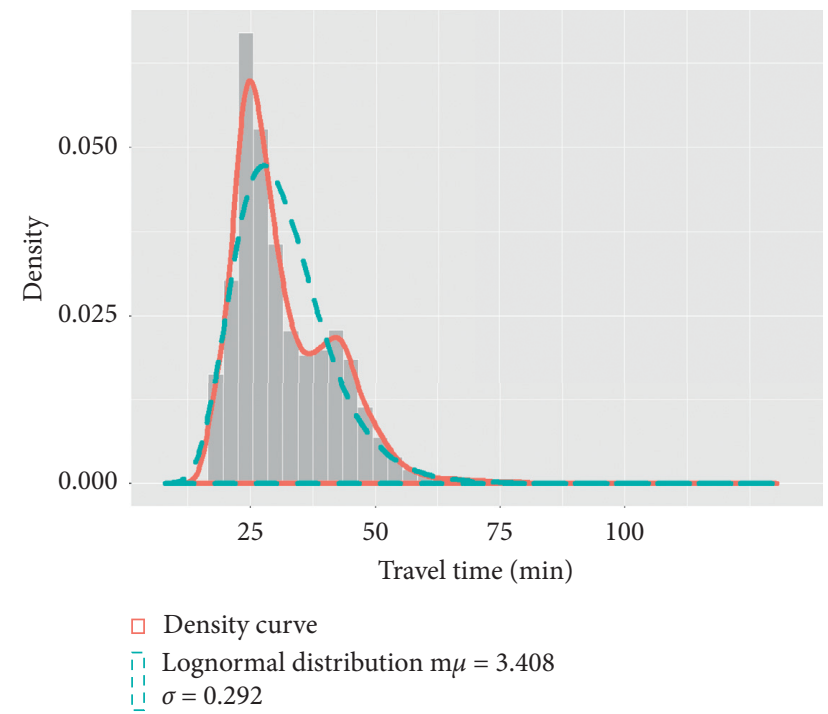

(d)

Figure 3: Selected distributions for the observed travel time data of four routes. (a) Yanan elevated expressway Eastbound, (b) Yanan elevated expressway Westbound. (c) Yanan arterial road Eastbound, and (d) Yanan arterial road Westbound.

Yanan arterial road have obviously higher values than those of eastbound. The BTIs (and MIs) of these two routes are $0.56(0.50)$ and $0.34(0.32)$, respectively. The possible reason for this is that tidal traffic phenomenon on Yanan arterial road westbound can result in more frequent traffic congestions during afternoon peak hours.

4.3. Impact of Adverse Weather on TTR. The analysis results in Section 4.1 indicate that the travel time distribution characteristics can be different with the consideration of time period and highway type. In order to investigate the impacts of adverse weather on TTR under different conditions, four scenarios are studied in this paper:

(i) Scenario 1: the traffic on elevated expressway during off-peak hours (ii) Scenario 2: the traffic on elevated expressway during peak hours

(iii) Scenario 3: the traffic on urban arterial road during off-peak hours

(iv) Scenario 4: the traffic on urban arterial road during peak hours

For each scenario, the data are divided into two categories (adverse weather and normal weather) and four TTR measures are calculated. The results are shown in Figure 7, and there are three main findings. First, adverse weather has negative impacts on the TTR. For all scenarios, the values of four TTR measures all increase to some extent under adverse weather conditions. Second, travel times are less reliable during peak hours than that during off-peak hours. Third, the results suggest that the travel times are more reliable on elevated expressway. 


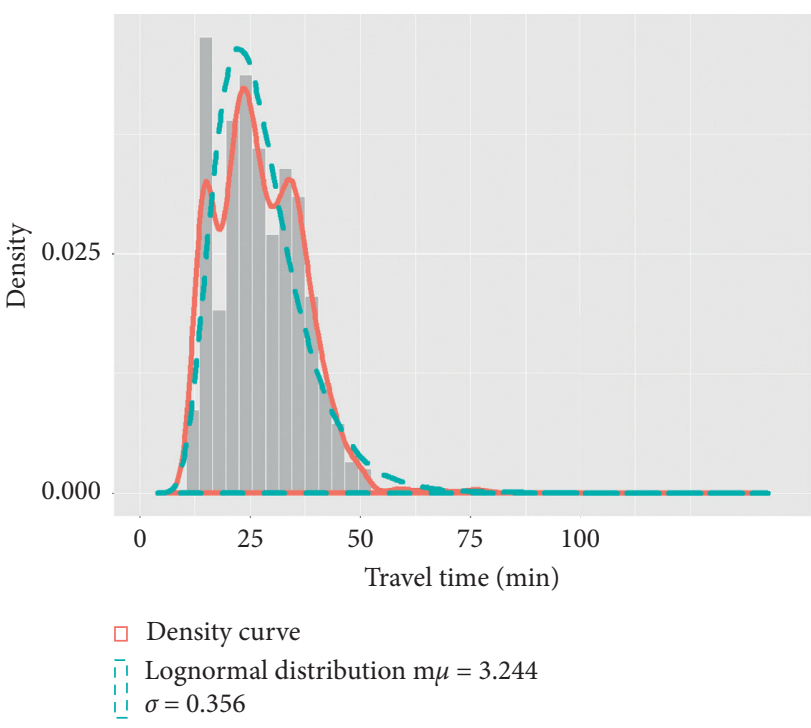

(a)

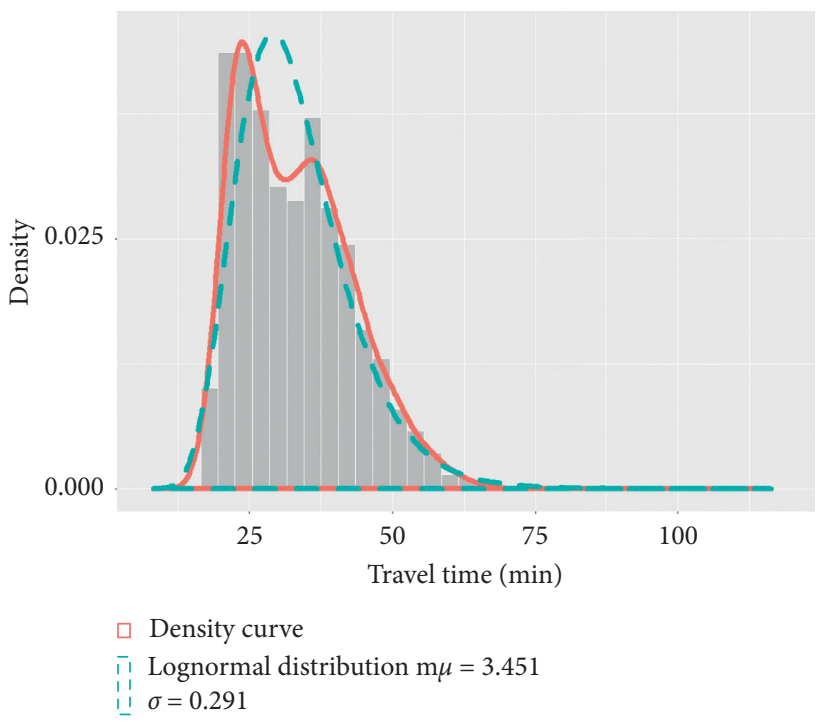

(c)

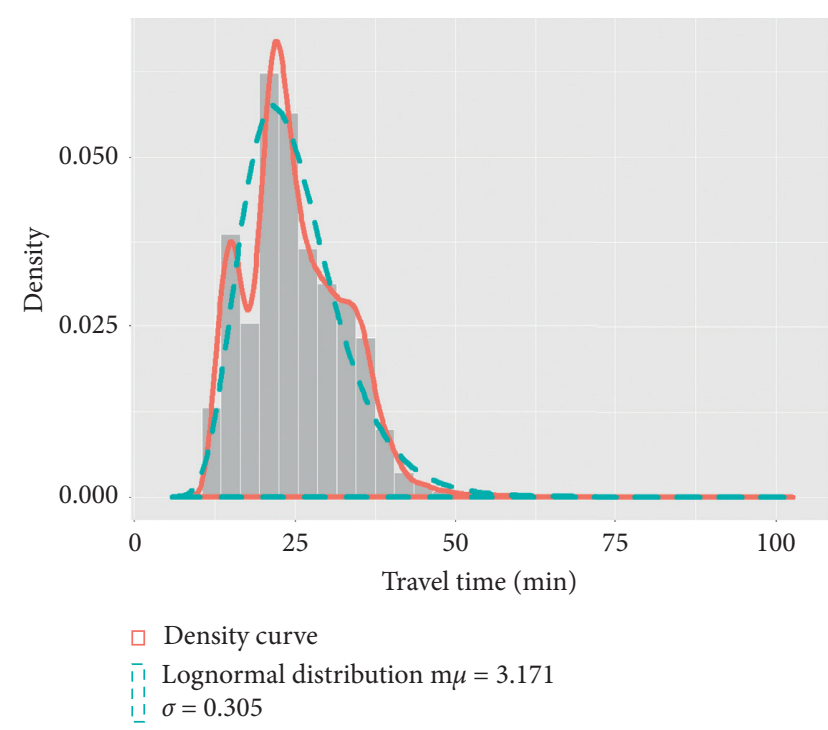

(b)

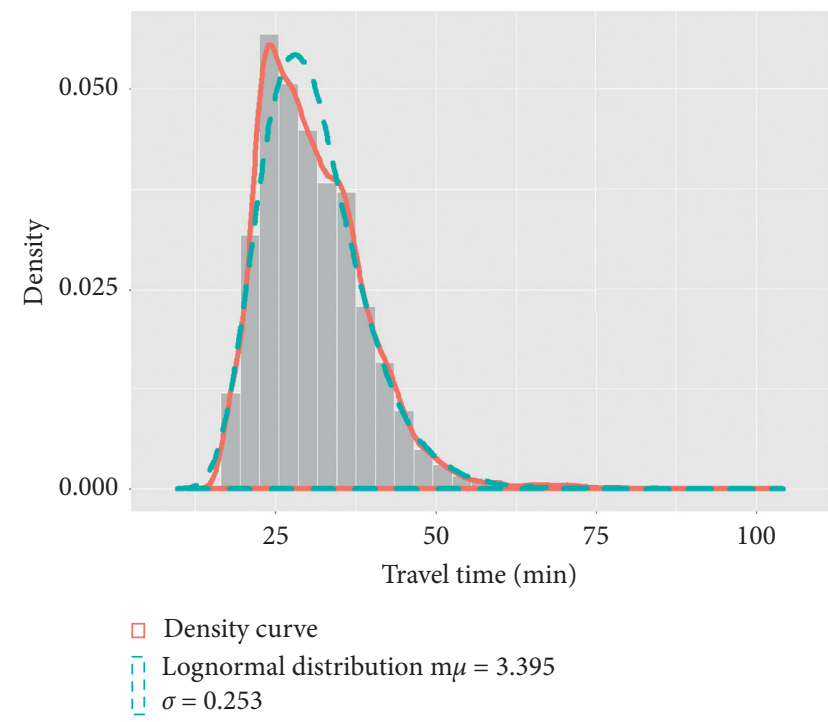

(d)

Figure 4: Selected distributions for the observed travel time data under different weather conditions. (a) Adverse weather of elevated expressway, (b) normal weather of elevated expressway, (c) adverse weather of urban arterial road, and (d) normal weather of urban arterial road.

Then, compared with the normal weather condition, the increase of TTR measures under adverse weather condition is calculated for each scenario. It is computed as the difference of TTR measure between two weather conditions divided by the TTR measure under normal weather conditions. The results on Table 3 show that, compared with offpeak hours, adverse weather has larger influences on TTR during peak hours. In the latter case, travel time variability under adverse conditions increased by an average of $29 \%$ when compared with normal conditions, while the buffer time index, misery index, and frequency of congestion increased by an average of $19 \%, 22 \%$, and $63 \%$, respectively. The possible reason is that the volume of traffic during peak hours is high, and, therefore, traffic collisions and congestions are more likely to occur during adverse weather.
4.4. Impact of Adverse Weather on Travel Delay. This section calculates the travel time delay under different conditions. The average delay was computed by subtracting the calculated travel time from the free-flow travel time for each four minutes period. As mentioned before, the free-flow travel time is defined as the $15^{\text {th }}$ percentile of travel time during overnight hours. The results are displayed in Figure 8(a). It is apparent that the adverse weather causes an overall larger travel delay. The possible explanation is that the adverse weather can result in traffic congestion. Note that the difference of the average travel delay between adverse weather and normal weather during off-peak period is not very noticeable but is remarkable during peak period. This can be explained by the fact that adverse weather may result in higher rates of the traffic congestion at higher inflow levels. 


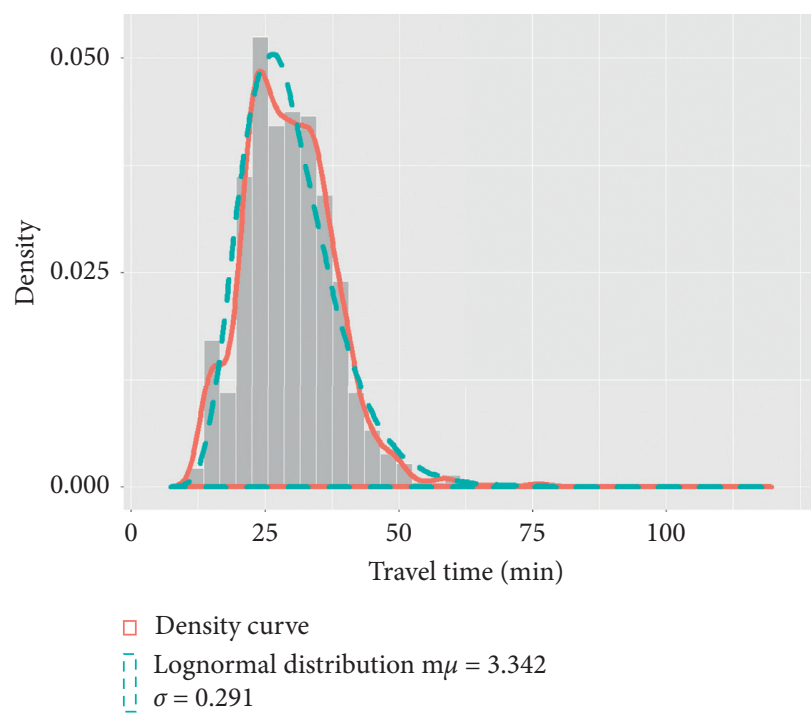

(a)

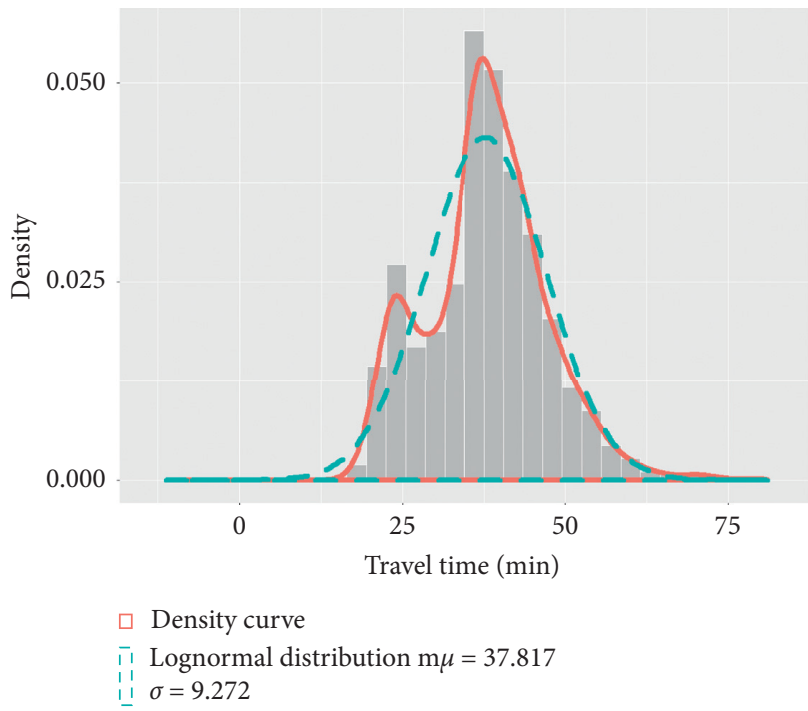

(c)

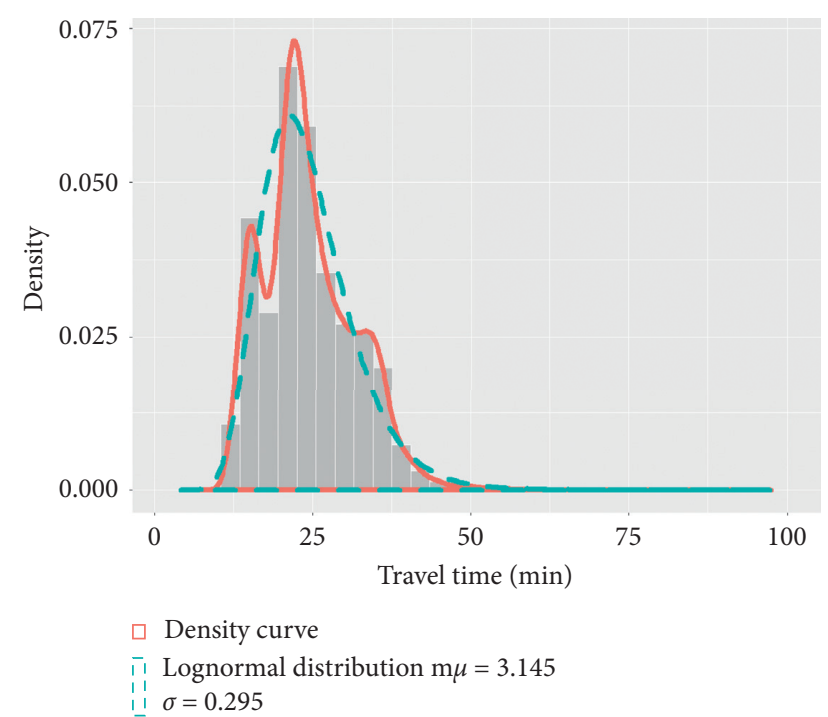

(b)

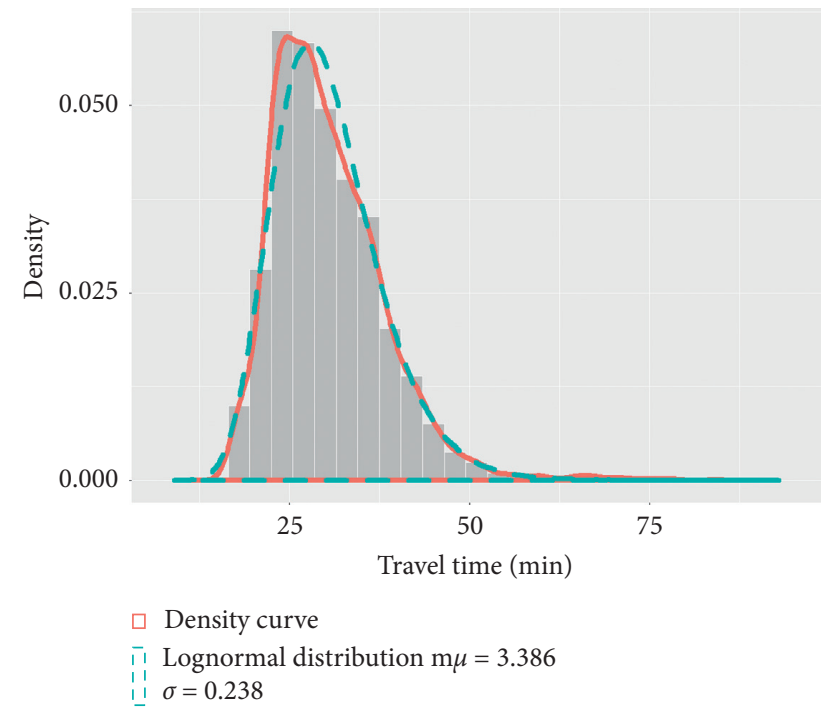

(d)

Figure 5: Selected distributions for the observed travel time data during different time periods. (a) Peak hours of elevated expressway, (b) off-peak hours of elevated expressway, (c) peak hours of urban arterial road, and (d) off-peak hours of urban arterial road.

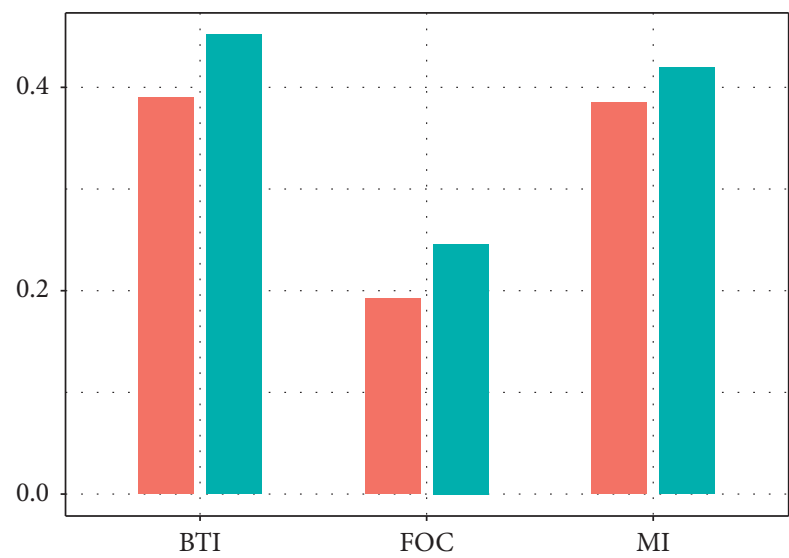

Eastbound

- Westbound

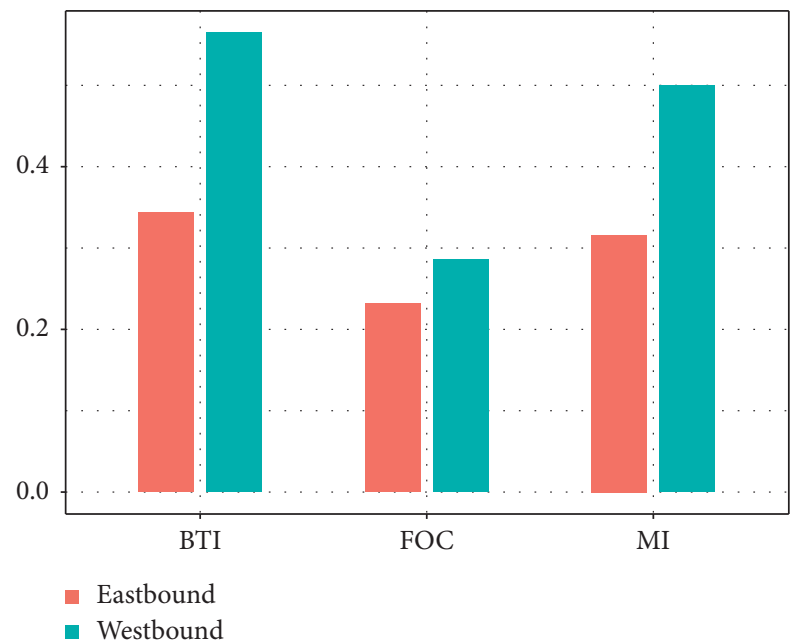

(b)

FIgURe 6: Buffer time index, misery index, and frequency of congestion of four routes. (a) Elevated expressway and (b) urban arterial road. 


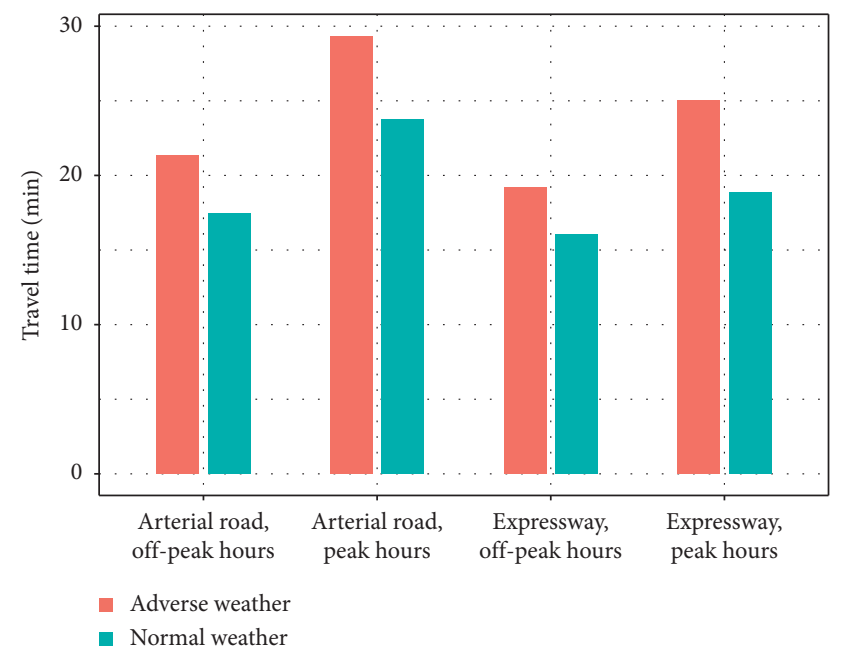

(a)

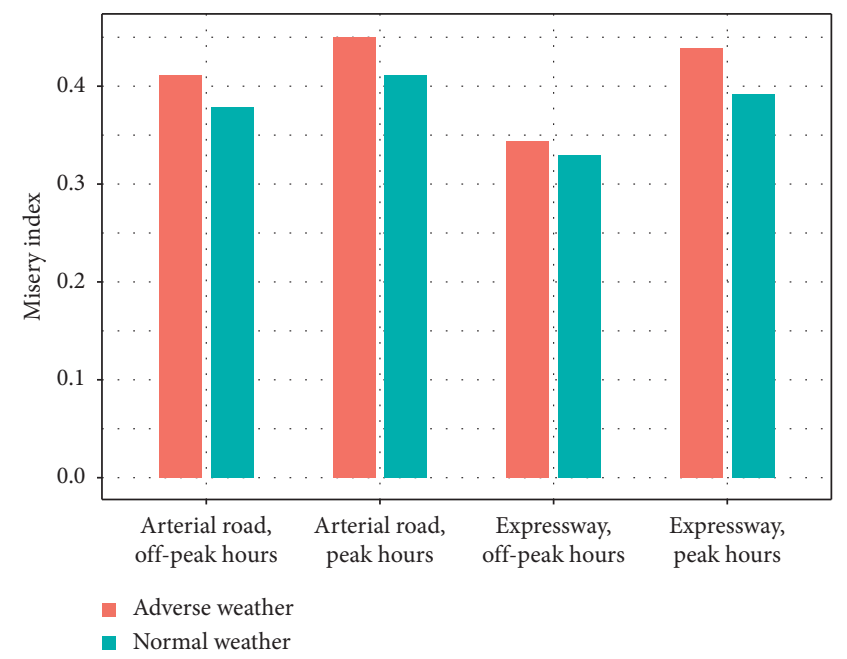

(c)

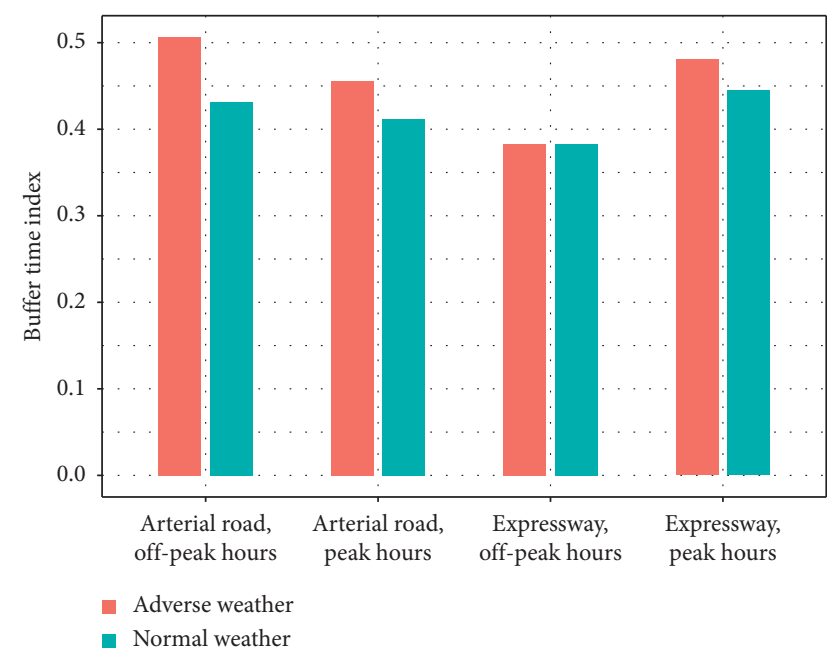

(b)

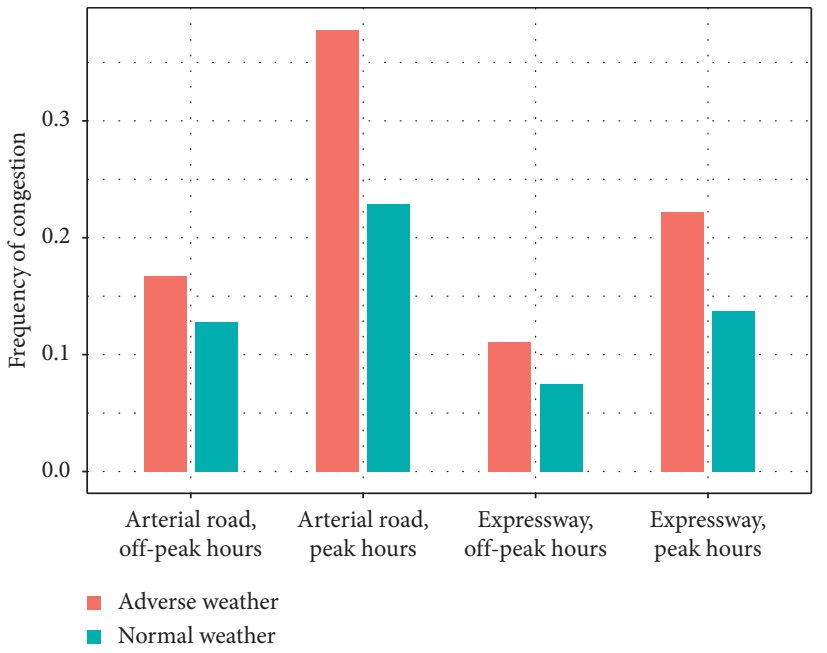

(d)

Figure 7: TTV, BTI, MI, and FOC under different scenarios. (a) TTV, (b) buffer time index, (c) misery index, and (d) frequency of congestion.

TABLE 3: The increase of TTR measures under adverse weather condition.

\begin{tabular}{|c|c|c|c|c|c|}
\hline Roadway type & Time & TTV (\%) & BTI (\%) & MI (\%) & FOC (\%) \\
\hline \multirow{2}{*}{ Elevated expressway } & Peak hours & 33 & 8 & 12 & 61 \\
\hline & Off-peak hours & 21 & 8 & 4 & 49 \\
\hline \multirow{2}{*}{ Urban arterial road } & Peak hours & 24 & 30 & 31 & 65 \\
\hline & Off-peak hours & 22 & 17 & 9 & 31 \\
\hline
\end{tabular}

The average delay time rate is defined as the ratio of the average delay to free-flow travel time in this paper. The results are summarized in Figure 8(b) and show that, during off-peak hours, the average delay for adverse weather increases by $6-9 \%$ compared to normal weather. The average delay increases by $18-30 \%$ during peak hours. Meanwhile, the average delay time rate of elevated expressway is larger than that of the arterial road under the same traffic and weather condition. Due to the speed limit and intersections, the free-flow travel time of arterial road is higher. 


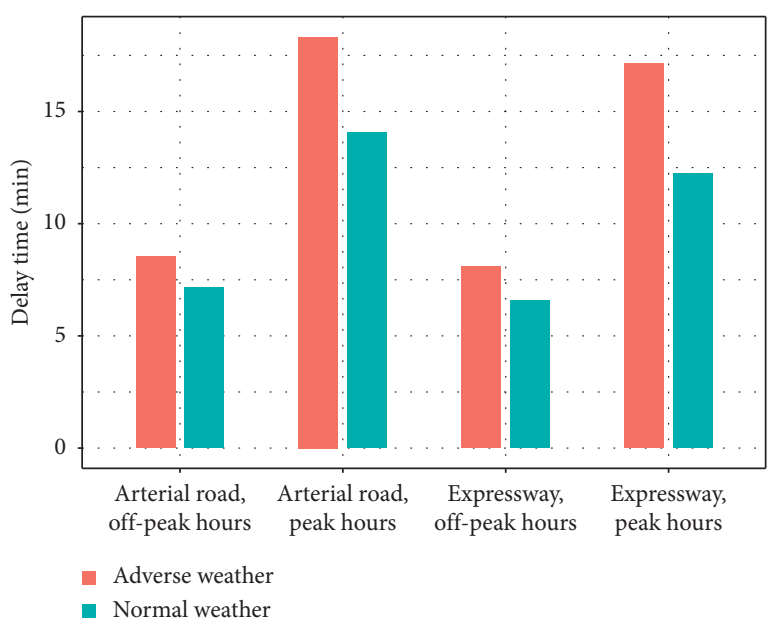

(a)

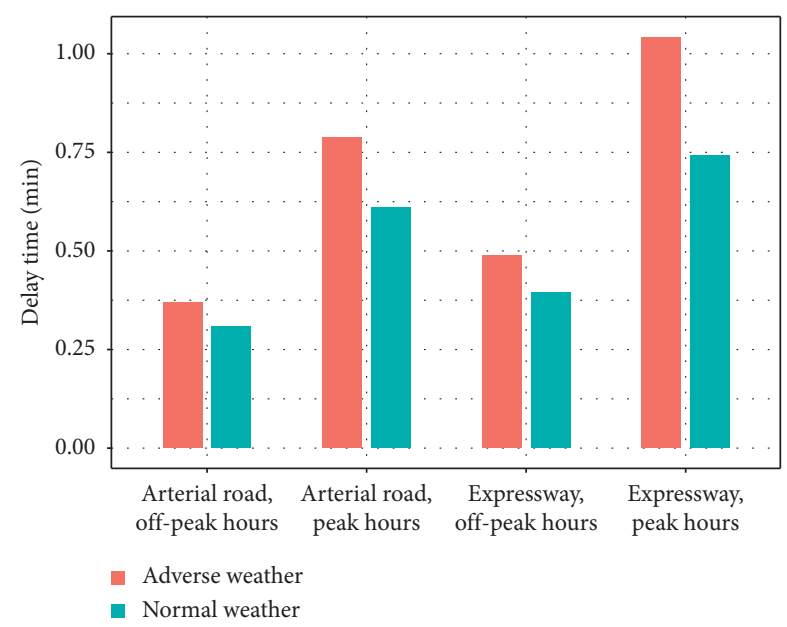

(b)

FIgURE 8: Travel delay under each condition. (a) Average delay and (b) average delay time rate.

\section{Conclusions}

In order to investigate the impact of adverse weather on travel time reliability of urban corridor under different conditions, this paper conducted an empirical study by using traffic data and weather data collected on Yanan corridor. Four typical scenarios with different highway types and time periods were studied in this paper, and the impact of adverse weather on urban corridor TTR under each scenario was evaluated against normal weather conditions. In addition, this study explored the distribution characteristics of travel time on urban roads with respect to the roadway type, time of day, and weather. Hence, the findings in this study are useful for determining traffic control strategies to address the adverse weather-related traffic congestions. And the distribution fitting results are useful for predicting travel time. The main findings can be summarized as follows.

(1) Among five widely used single-mode distribution types (i.e., Weibull, Gamma, Normal, Lognormal, and Log-logistic), the Lognormal distribution outperforms other models for most conditions, except the urban arterial road data observed during peak hours.

(2) Adverse weather clearly shows negative impacts on the travel time reliability of urban corridor, and the magnitude of the effects can be different under different scenarios. Compared to off-peak hours, adverse weather has larger influences on TTR during peak hours.

(3) During peak hours, travel time variability under adverse weather conditions increased by an average of $29 \%$ when compared to normal conditions, while the buffer time index, misery index and frequency of congestion increased by an average of $19 \%, 22 \%$, and $63 \%$, respectively.

For future work, the choice of distribution types can be expanded when analysing the travel time distribution, since the multimode distribution can provide a better fitting performance under some traffic conditions [34-37]. This paper mainly examined the difference of rainy weather's impact on TTR under different conditions; thus, the difference of other adverse weather should also be investigated if the sample size for each category is sufficient. In addition, the traffic composition and traffic flow rate should also be further considered in the future study.

\section{Data Availability}

The data used to support the findings of this study are available from the corresponding author upon request.

\section{Conflicts of Interest}

The authors declare that they have no conflicts of interest.

\section{Acknowledgments}

This research was sponsored jointly by the National Key Research and Development Program of China (Grant no. 2018YFE0102800) and the Fundamental Research Funds for the Central Universities (grant no. 22120200035).

\section{References}

[1] D. Fha, Travel Time Reliability: Making it There on Time, All the Time, US Department of Transportation, Federal Highway Administration, Washington, NJ, USA, 2010.

[2] M. Abdel-Aty, R. Kitamura, and P. P. Jovanis, "Investigating effect of travel time variability on route choice using repeatedmeasurement stated preference data," Transportation Research Record, vol. 1493, pp. 39-45, 1995.

[3] Z. Li, D. A. Hensher, and J. M. Rose, "Willingness to pay for travel time reliability in passenger transport: a review and some new empirical evidence," Transportation Research Part E: Logistics and Transportation Review, vol. 46, no. 3, pp. 384-403, 2010.

[4] C. Chen, A. Skabardonis, and P. Varaiya, "Travel-time reliability as a measure of service," Transportation Research Record: Journal of the Transportation Research Board, vol. 1855, no. 1, pp. 74-79, 2003. 
[5] T. C. Lam, The Effect of Variability of Travel Time on Route and Time-Of-Day Choice, University of California, Oakland, CA, USA, 2000.

[6] Q. Shi and M. Abdel-Aty, "Evaluation of the impact of travel time reliability on urban expressway traffic safety," Transportation Research Record: Journal of the Transportation Research Board, vol. 2582, no. 1, pp. 8-17, 2016.

[7] E. Kidando, R. Moses, E. E. Ozguven, and T. Sando, "Evaluating traffic congestion using the traffic occupancy and speed distribution relationship: an application of Bayesian Dirichlet process mixtures of generalized linear model," Journal of Transportation Technologies, vol. 7, no. 3, p. 318, 2017.

[8] R. J. Javid and R. J. Javid, "A framework for travel time variability analysis using urban traffic incident data," IATSS Research, vol. 42, no. 1, pp. 30-38, 2018.

[9] W. Fan and L. Gong, "Developing a Systematic Approach to Improving Bottleneck Analysis in North Carolina," FHWA/ NC/2016-10, North Carolina Dept. of Transportation, Raleigh, NC, USA, 2017.

[10] A. T. Hojati, L. Ferreira, S. Washington, P. Charles, and A. Shobeirinejad, "Reprint of: modelling the impact of traffic incidents on travel time reliability," Transportation Research Part C: Emerging Technologies, vol. 70, pp. 86-97, 2016.

[11] M. Figliozzi and A. Bigazzi, Value of Travel-Time Reliability, Part II: A Study of Tradeoffs between Travel Reliability, Congestion-Mitigation Strategies and Emissions, Oregon Transportation Research and Education Consortium, Portland, OR, USA, 2012.

[12] F. Yang, M.-P. Yun, and X.-G. Yang, "Travel time distribution under interrupted flow and application to travel time reliability," Transportation Research Record: Journal of the Transportation Research Board, vol. 2466, no. 1, pp. 114-124, 2014.

[13] T. Lomax, D. Schrank, S. Turner, and R. Margiotta, Report of Selecting Travel Reliability Measures, Federal Highway Administration, Washington, NJ, USA, 2003.

[14] W. Pu, "Analytic relationships between travel time reliability measures," Transportation Research Record: Journal of the Transportation Research Board, vol. 2254, no. 1, pp. 122-130, 2011.

[15] H. Tu, H. W. Van Lint, and H. J. Van Zuylen, "Impact of adverse weather on travel time variability of freeway corridors," in Proceedings of the Transportation Research Board 86th Annual Meeting, Washington, NJ, USA, January 2007.

[16] J. W. C. Van Lint and H. J. van Zuylen, "Monitoring and predicting freeway travel time reliability: using width and skew of day-to-day travel time distribution," Transportation Research Record: Journal Transportation Research Record, vol. 1917, no. 1, pp. 54-62, 2005.

[17] P. Chen, R. Tong, G. Lu, and Y. Wang, "Exploring travel time distribution and variability patterns using probe vehicle data: case study in Beijing," Journal of Advanced Transportation, vol. 2018, Article ID 3747632, 13 pages, 2018.

[18] L. Gao, Research on Reliability of Path Travel Time Based on Dynamic Travel Time, Hebei University of Technology, Tianjin, China, 2011.

[19] E. Milliken, R. Young, and M. P. Consortium, Use of Travel Time, Travel Time Reliability, and Winter Condition Index Information for Improved Operation of Rural Interstates, Mountain Plains Consortium, Fargo, ND, USA, 2015.

[20] B. Yao, P. Hu, X. Lu, J. Gao, and M. Zhang, "Transit network design based on travel time reliability," Transportation Research Part C: Emerging Technologies, vol. 43, pp. 233-248, 2014.
[21] S. Mathew and S. S. Pulugurtha, "Assessing the effect of a light rail transit system on road traffic travel time reliability," Public Transport, vol. 12, pp. 1-21, 2020.

[22] K. Lyman and R. L. Bertini, "Using travel time reliability measures to improve regional transportation planning and operations," Transportation Research Record, vol. 2046, no. 1, pp. 1-10, 2008.

[23] M. G. Cedillo-Campos, C. M. Pérez-González, J. Piña-Barcena, and E. Moreno-Quintero, "Measurement of travel time reliability of road transportation using GPS data: a freight fluidity approach," Transportation Research Part A: Policy and Practice, vol. 130, pp. 240-288, 2019.

[24] M. A. P. Taylor, "Dense network traffic models, travel time reliability and traffic management. II: application to network reliability," Journal of Advanced Transportation, vol. 33, no. 2, pp. 235-251, 1999.

[25] Z. Li, L. Elefteriadou, and A. Kondyli, "Quantifying weather impacts on traffic operations for implementation into a travel time reliability model," Transportation Letters, vol. 8, no. 1, pp. 47-59, 2016.

[26] I. Tsapakis, T. Cheng, and A. Bolbol, "Impact of weather conditions on macroscopic urban travel times," Journal of Transport Geography, vol. 28, pp. 204-211, 2013.

[27] M. A. Yazici, C. N. Kamga, and A. Singhal, "Weather's impact on travel time and travel time variability in New York city," Transportation Research, vol. 40, p. 41, 2013.

[28] K. P. Burnham and D. R. Anderson, "Multimodel inference," Sociological Methods \& Research, vol. 33, no. 2, pp. 261-304, 2004.

[29] J. W. C. Van Lint, H. J. Van Zuylen, and H. Tu, “Travel time unreliability on freeways: why measures based on variance tell only half the story," Transportation Research Part A: Policy and Practice, vol. 42, no. 1, pp. 258-277, 2008.

[30] H. Tu, H. Li, H. Van Lint, and H. Van Zuylen, "Modeling travel time reliability of freeways using risk assessment techniques," Transportation Research Part A: Policy and Practice, vol. 46, no. 10, pp. 1528-1540, 2012.

[31] Z. Chen and W. Fan, "Data analytics approach for travel time reliability pattern analysis and prediction," Journal of Modern Transportation, vol. 27, no. 4, pp. 250-265, 2019.

[32] D. Schrank, B. Eisele, T. Lomax, and J. Bak, Urban Mobility Scorecard, The Texas A\&M Transportation Institute and The Texas A\&M University System, College Station, TX, USA, 2015.

[33] B. L. Smith and J. M. Ulmer, "Freeway traffic flow rate measurement: investigation into impact of measurement time interval," Journal of Transportation Engineering, vol. 129, no. 3, pp. 223-229, 2003.

[34] S. Park, H. Rakha, and F. Guo, "Calibration issues for multistate model of travel time reliability," Transportation Research Record: Journal of the Transportation Research Board, vol. 2188, no. 1, pp. 74-84, 2010.

[35] H. Rakha, I. El-Shawarby, and M. Arafeh, "Trip travel-time reliability: issues and proposed solutions," Journal of Intelligent Transportation Systems, vol. 14, no. 4, pp. 232-250, 2010.

[36] X. Zhong, Y. Zou, Z. Dong, S. Yuan, and M. Ijaz, "Finite mixture survival model for examining the variability of urban arterial travel time for buses, passenger cars and taxis," IET Intelligent Transport Systems, vol. 45, pp. 1524-1533, 2020.

[37] L. Wang, H. Zhong, W. Ma, M. Abdel-Aty, and J. Park, "How many crashes can connected vehicle and automated vehicle technologies prevent: a meta-analysis," Accident Analysis \& Prevention, vol. 136, Article ID 105299, 2020. 